\title{
Involvement of semicarbazide-sensitive amine oxidase-mediated deamination in atherogenesis in KKAy diabetic mice fed with high cholesterol diet
}

\author{
P. H. Yu, M. Wang, Y. L. Deng, H. Fan, L. Shira-Bock \\ Neuropsychiatry Research Unit, University of Saskatchewan, Saskatoon, Saskatchewan, Canada
}

\begin{abstract}
Aims/hypothesis. Semicarbazide-sensitive amine oxidase has been recognised to be a potential risk factor in vascular disorders associated with diabetic complications and to be related to mortality in patients suffering from heart disease. This enzyme, associated with the vascular system, catalyses the deamination of methylamine and aminoacetone, and also acts as an adhesion molecule related to leucocyte trafficking and inflammation. The deaminated products include the toxic aldehydes, formaldehyde and methylglyoxal, respectively, hydrogen peroxide and ammonia.

Materials and methods. In this study, the KKAy mouse, a strain possessing features closely resembling those of Type II (non-insulin-dependent) diabetes mellitus has been used to substantiate the hypothesis. Vascular lesions were induced via chronic feeding of a high cholesterol diet.

Results. Both MDL-72974A, a selective mechanismbased semicarbazide-sensitive amine oxidase inhibitor
\end{abstract}

and aminoguanidine effectively inhibited aorta semicarbazide-sensitive amine oxidase activity, and caused a substantial increase in urinary methylamine, and a decrease in formaldehyde and methylgloxal levels. Inhibition of semicarbazide-sensitive amine oxidase also reduced oxidative stress, as shown by a reduction of malondialdehyde excretion. Both MDL-72974A and aminoguanidine reduced albuminuria, proteinuria and the number of atherosclerotic lesions in animals fed with a cholesterol diet over a period of treatment for 16 weeks.

Conclusion/interpretation. Increased semicarbazidesensitive amine oxidase-mediated deamination could be involved in the cascade of atherogenesis related to diabetic complications. [Diabetologia (2002) 45: 1255-1262]

Keywords Methylamine, formaldehyde, methylglyoxal, aminoguanidine, semicarbazide-sensitive amine oxidase (SSAO), SSAO inhibitor, atherosclerosis.

Serum semicarbazide-sensitive amine oxidase (SSAO) activity has been repeatedly found to be increased in diabetic patients $[1,2,3]$ and in diabetic animals [4, 5]. Of interest, serum SSAO is positively correlated with retinopathy in diabetic patients [6]. Increased serum SSAO has also been detected in patients with heart failure [7] and cerebral infarct [8] and in atherosclerotic as well as in obese subjects [9]. The mortality of heart patients with high serum SSAO activity was found to be higher than in heart patients with low serum SSAO levels $[10,11]$.

SSAO is primarily located on the plasma membrane in endothelial and vascular smooth muscle cells, and adipose and cartilage tissues [12]. It also circu- 
lates in the blood. SSAO catalyses the deamination of methylamine and aminoacetone to produce toxic formaldehyde and methylglyoxal, respectively [13, $14,15]$. These highly reactive aldehydes can initiate unwanted protein cross-linkage, exacerbate advanced glycation end products, and cause endothelial injury [16]. While deamination of methylamine causes toxicity towards cultured human endothelial cells, SSAO inhibitors have been shown to prevent this toxicity [17]. Chronic methylamine treatment increased oxidative stress, and possibly vascular damage, as indicated by the increase of urinary excretion of malondialdehyde in rats [18]. It has also been shown that ${ }^{14} \mathrm{C}$ methylamine is converted to ${ }^{14} \mathrm{C}$-formaldehyde, which then form radioactively labelled long-lasting protein adducts in vivo [19]. Aminoguanidine, which is known to block advanced glycation and reduce nephropathy in animals, is a much more potent inhibitor of SSAO [20]. Inhibition of SSAO by aminoguanidine could also be involved in preventing nephropathy. A selective SSAO inhibitor has been shown to reduce the apparent nephropathy in STZ-induced diabetic rats and in methylamine-treated mice [19, 20]. Methylamine is a major metabolite of creatine [21]. It can also be derived from adrenaline, lecithin, or food and cigarette smoke. Increased deamination of methylamine has been proposed to be related to nephropathy [22].

The KKAy mouse strain possesses features closely resembling those of Type II (non-insulin-dependent) diabetes mellitus and serves as a useful model for the study of obesity and diabetes [23, 24]. It is derived from a Japanese KK mouse strain incorporating a yellow agoutis gene $(A y)$ [25]. KKAy mice are characterised by severe and prolonged hyperinsulinaemia, hyperglycaemia and diabetic complications [26]. They spontaneously develop Type II diabetes during the first 8 weeks of life. These animals were used in this study to test the hypothesis that SSAO-mediated deamination is involved in vascular damage and atherogenesis. The effects of chronic administration of the SSAO inhibitors (aminoguanidine and MDL-72974A) on albuminuria and atherosclerotic lesions in these mice fed a cholesterol-rich diet were assessed.

\section{Materials and methods}

Materials. $[7-14 \mathrm{C}]$-Benzylamine hydrochloride $(59 \mathrm{mCi} / \mathrm{mmol})$ was purchased from Amersham Life Science (Amersham International, Buckinghamshire, UK). MDL-72974A was kindly provided by Marion-Merrell-Dow (Cincinnati, Ohio, USA). All other chemicals are of analytical grade.

Animal experiments. Female KKAy mice were obtained from Clea (Tokyo, Japan). The animal studies were in strict accordance with the guidelines established by the Canadian Council on Animal Care and were approved by the University of Saskatchewan Animal Care Committee. The mice were housed in hanging wire cages with free access to food and water on a 12-h light to dark cycle at a temperature of 19 to $20^{\circ} \mathrm{C}$.

The KKAy mice at the age of 9 weeks with confirmed diabetes were randomly divided into five groups ( $n=16$ in each group). These are (i) control, normal diet; (ii) MDL-72974A treatment ( $2 \mathrm{mg}$ per $\mathrm{kg}$ per day via drinking water); (iii) aminoguanidine treatment (50 mg per $\mathrm{kg}$ per day); (iv) control, atherogenic diet; (v) atherogenic diet plus MDL-72974A.

The high cholesterol diet was obtained from ICN Pharmaceuticals (Aurora, Ohio, USA). The diet contained 1.25\% cholesterol, $7.5 \%$ cocoa butter, $5 \%$ sodium cholate, and other sugar and minerals.

Urine collection. The mice were placed in metabolic cages (Nalgene, Rochester, N.Y., USA) for urine collection over a period of $20 \mathrm{~h}$. The urine collecting vessels were positioned over Styrofoam containers filled with dry ice, which permitted the freezing of urine immediately after urination. The animals were allowed free access to tap water. During urine collection, food was withheld.

SSAO activity assay. SSAO activity was assessed by a radioenzymatic procedure using ${ }^{14} \mathrm{C}$-labelled benzylamine as substrate following our previously described procedure [17]. Aortae were dissected and kept frozen at $-70^{\circ} \mathrm{C}$. The tissues were then homogenized with a Polytron homogenizer (Kinematica, Lucerne, Switzerland) followed by brief sonication before assessment of SSAO activity.

Analysis of methylamine. Urinary methylamine was measured using an HPLC-fluorometric procedure [20]. The urine samples, following purification using a small CG-50 amberlite column, were pre-column derivatized with o-phthaldialdehyde (OPA) and then applied to a Beckman Ultrasphere IP column (octadecyl-bonded spherical-5 micron silica particles) using a Shimadzu HPLC system (Sil-9A auto injector) equipped with a pre-column derivatization program. The column was eluted with $65 \%$ methanol at a flow rate of $1.0 \mathrm{ml} / \mathrm{min}$. For quantitative assessment, a programmable fluorescence detector (Hewlett Packard, HP1046A) with excitation at $360 \mathrm{~nm}$ and emission at $445 \mathrm{~nm}$ was used. Isopropylamine was used as an internal standard.

Analysis of urinary aldehydes. A HPLC-spectro-photometric method was used for the assessment of aldehydes [27]. Aldehydes were derivatized with 2.4-dinitrophenyl-hydrazine (DNPH). Propionaldehyde was used as an internal standard. The HPLC separation was carried out using a reversed-phase Ultrasphere I.P. analytical column $(250 \times 4.6 \mathrm{~mm}$ I.D., Beckman, Toronto, Canada). Elution was isocratic with $20 \mathrm{mmol} / \mathrm{l}$ phosphate buffer, $\mathrm{pH} 4.6$, containing $32 \%$ acetonitrile and $8 \%$ 2 -methylpropanol at a flow rate of $1.0 \mathrm{ml} / \mathrm{min}$. The DNPH derivatives were analysed via a spectro-detector at $330 \mathrm{~nm}$.

Assessment of urinary creatine and creatinine. Creatinine was assessed using Jaffe's reaction, which results in the production of a red tautomer of creatinine picrate after adding an alkaline picrate solution [28], which was measured at $520 \mathrm{~nm}$. Creatine was estimated as the difference between the preformed creatinine and the total creatinine that results after the conversion: creatine vs creatinine by heating at an acid $\mathrm{pH}$.

ELISA for the detection of mouse albumin. This method is in principle based on a method described previously [29]. Antibodies, i.e. rabbit anti-mouse albumin; Goat anti-mouse albumin conjugated with peroxidase, made by Biogenesis were purchased from Cedarlane. Plastic 96-well EIAr plates were 
coated with polyclonal rabbit anti-mouse albumin antibodies followed by coating with skimmed milk. Urine samples and standard spiking mouse albumin were added and plates were washed thoroughly. Polyclonal goat anti-mouse albumin antibody conjugated with peroxidase was then added and incubated for $1 \mathrm{~h}$ at room temperature. After three washes with PBS o-phenylenediamine dihydrochloride (OPD) substrate solution [one tablet OPD $(10 \mathrm{mg})$ and $6 \mu \mathrm{l} 50 \% \mathrm{H}_{2} \mathrm{O}_{2}$ in $25 \mathrm{ml}$ of buffer] were added and incubated for $20 \mathrm{~min}$ at $37^{\circ} \mathrm{C}$. To stop reactions $25 \mu \mathrm{l} 3 \mathrm{~N} \mathrm{HCl}$ were added. Optical densities at $490 \mathrm{~nm}$ were measured using an automatic ELISA reader. Urinary albumin was estimated from an albumin standard curve. Urinary protein was calculated by the Bradford method.

Atherosclerotic lesions. The heart and attached section of aorta were placed in $0.9 \%$ saline for $1 \mathrm{~h}$, then fixed in $4 \%$ formaldehyde and embedded in OCT. For quantitative evaluation of atherosclerotic lesions the hearts along with the aortae were frozen on a cryostat and then sectioned at a thickness of $10 \mu \mathrm{m}$. The region of the aortae to be sectioned began at the juncture of the aorta to the heart and continued towards the aortic arch for a distance of approximately $350 \mu \mathrm{m}$. Every second $10 \mu \mathrm{m}$ section was collected, fixed on gelatin-coated microscope slides, and then stained with Oil Red $\mathrm{O}$ and haematoxylin. The numbers of lesions were counted by examining the lipid deposits in the intima [30].

Statistics. The results were assessed using ANOVA followed by Newman-Keuls multiple comparisons. In general, the null hypothesis used for all analyses was that the factor has no influence on the measured variable and significance was accepted at a confidence interval of more than $95 \%$.

\section{Results}

Hyperglycaemia of the KKAy mice. Serum blood glucose concentrations of all KKAy mice were assessed. Venous blood was collected from the tails. Blood glucose concentrations were over $14 \mathrm{mmol} / \mathrm{l}$ in 89 of 97 mice at 9-weeks old (before drug treatment). The remaining 8 mice previously shown to be normal also became hyperglycaemic on week 15 (6 weeks after the first glucose assessment). The blood glucose concentrations of the non-diabetic CD-1 Swiss White mice ranged from 6 to $9 \mathrm{mmol} / \mathrm{l}$. All of the KKAy mice became hyperglycaemic.

SSAO activity in KKAy mice. The KKAy mice showed


$7 \mathrm{pmol} \cdot \mathrm{mg}$ protein ${ }^{-1} \cdot \mathrm{min}^{-1}$ ) in comparison to that of Swiss White CD-1 mice $(35 \pm 4$ pmol.mg protein $^{-1} \cdot \mathrm{min}^{-1}$ ) from a parallel assessment. The data represent the mean \pm SEM $(n=5)$.

Effect of MDL-72974A on KKAy SSAO activity. The inhibitory effect of MDL-72974A on KKAy aorta SSAO was assessed both in vitro and ex vivo. The $\mathrm{IC}_{50}\left(2 \times 10^{-8} \mathrm{~mol} / \mathrm{l}\right)$ values are quite similar to that compared with SSAO from other sources [31]. More than $90 \%$ inhibition of kidney and aorta SSAO activities was achieved after $24 \mathrm{~h}$ of treatment via drinking

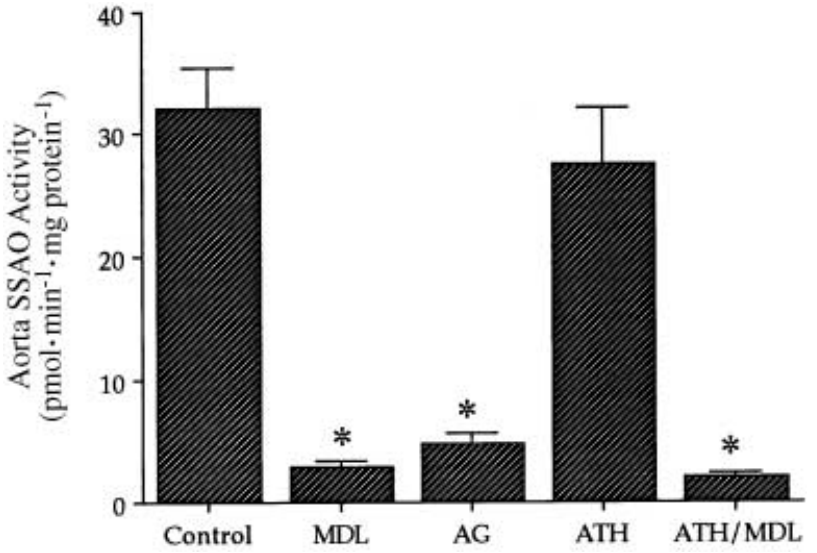

Fig. 1. Effect of MDL-72974A, aminoguanidine and atherogenic diet on KKAy aorta SSAO activities. Data represent means \pm SEM of eight animals. Significantly different from the control group $(* p<0.01)$

water containing MDL-72974A (10 $\mu \mathrm{g} / \mathrm{ml})$. Virtually a complete inhibition of SSAO activity was detected following a further 1 week of treatment via drinking water (a daily dose of approximately $2 \mathrm{mg}$ per $\mathrm{Kg}$ ). The aorta SSAO activities in the KKAy mice after 16 weeks of treatment are shown (Fig. 1). Although SSAO activity was initially completely inhibited by MDL-72974A and aminoguanidine, a low amount of SSAO activity (i.e. 6-15\%) was detected after treatment for 16 weeks. The effect of MDL-72974A treatment on kidney SSAO is quite comparable to that of the aorta.

Urinary methylamine. Urinary methylamine levels in the animals receiving an atherogenic diet were gradually reduced, while the methylamine excretion in mice treated with a normal diet was relatively constant over the period of treatment (Fig. 2B). We expected that inhibition of SSAO activity by MDL-72974A and aminoguanidine would cause a substantial increase in methylamine excretion compared with the corresponding controls for the duration of the experimental period. However, the initial increase in methylamine levels gradually decreased after 6 weeks of treatment.

Albuminuria and proteinuria. The results of the effects of administration of an atherogenic diet, with or without an SSAO inhibitor, on albuminuria over the 16-week treatment period are summarized (Fig. 3). Albuminuria increased with age in these Type II diabetic mice. A remarkable effect was seen in that MDL-72974A reduced the albumin in the animals fed an atherogenic diet (Fig. 3A). The effects of both MDL-72974A and aminoguanidine on albuminuria and proteinuria in animals fed a normal feed were less marked (Fig. 3B). Both MDL-72974A and aminoguanidine showed similar effects on the prevention of proteinuria. 

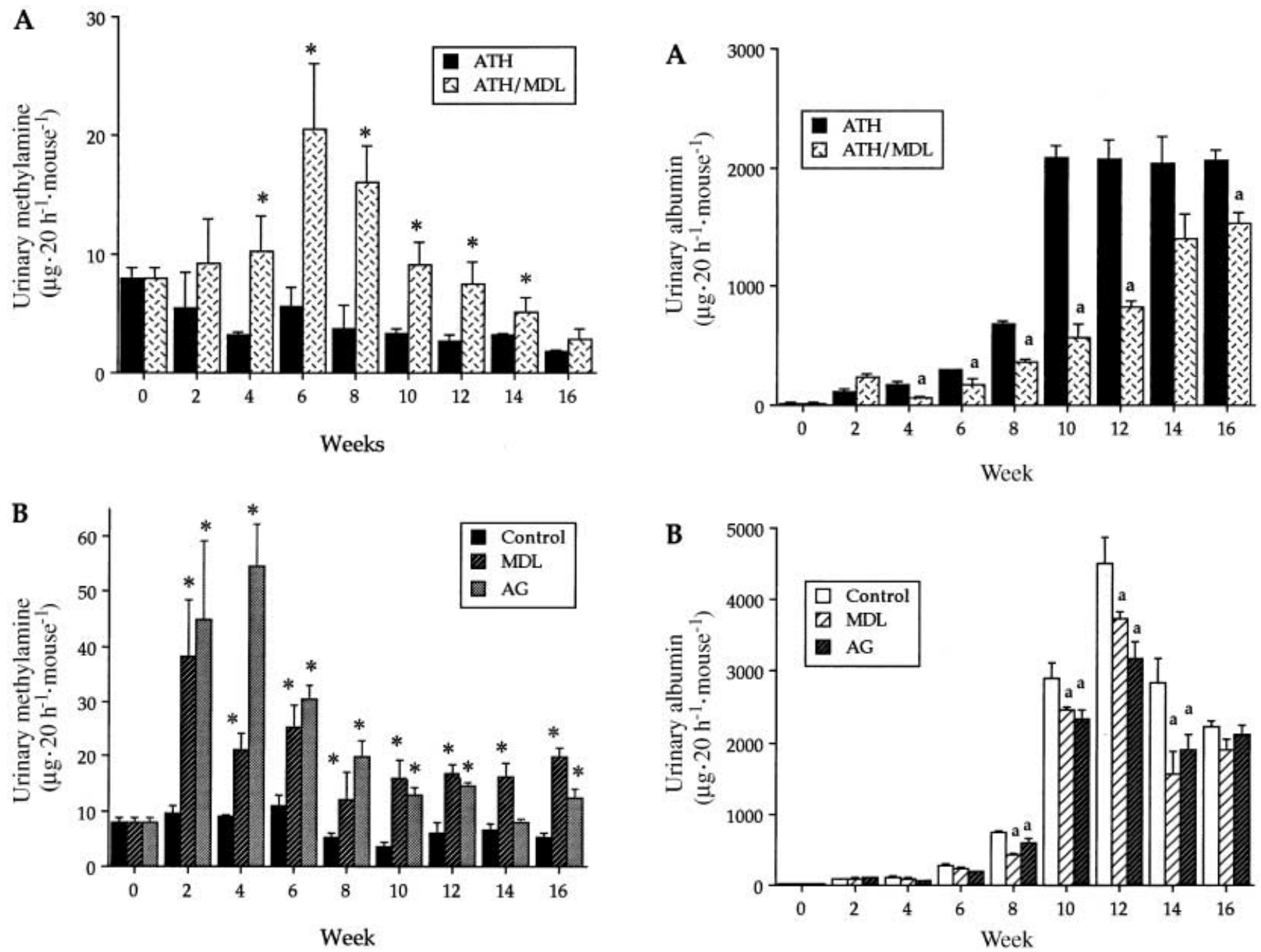

Fig. 2A, B. Effect of MDL-72974A, aminoguanidine and atherogenic diet on urinary methylamine levels from KKAy mice. Mice were fed with atherogenic diet (A) or normal diet (B). The urinary excretion of methylamine was monitored bi-weekly. Data represent means \pm SEM of eight mice. $* p<0.01$ in comparison to the corresponding control groups

Creatine. Urinary creatine, a major precursor for methylamine [32], was slightly increased with age in animals fed either a normal or an atherogenic diet. The increase in creatine levels with age seemed to be correlated to the increase in body mass. Neither MDL72974A nor aminoguanidine affected creatine excretion.

Urinary aldehydes. Urinary methylglyoxal (MGO), formaldehyde (FA) and malondialdehyde (MDA) were analysed 10 weeks after treatment regimen (Fig. 4). Both MDL-72974A and aminoguanidine not only reduced urinary formaldehyde and methylglyoxal levels, but also malondialdehyde excretion. An atherogenic diet caused an increase in urinary MDA, a marker for oxidative stress. Although MDA is not a product of SSAO-mediated deamination, its levels were reduced after treatment with SSAO inhibitors.

Fig. 3A, B. Effect of MDL-72974A, aminoguanidine and atherogenic diet on albuminuria in KKAy mice. Animals were fed with atherogenic diet (A) or normal diet (B). The urinary excretion of albumin was monitored bi-weekly. Data represent means \pm SEM of eight mice. ${ }^{*} p<0.01$ in comparison to the control group with atherogenic diet

Atherosclerotic lesions. Eight mice from experimental groups, i.e. (1) (2) and (3) were killed at week 6. The rest of the experimental mice in all five groups ( 8 to 9 mice) were killed after 16 weeks. Atherosclerotic lesions were microscopically assessed. At week 6 , no fatty streaks were found in any of the experimental groups. After 16 weeks treatment, fatty streaks of various sizes were observed on the aortae. Lesions were shown primarily in two distinct locations, namely the aortic wall and valve cusp. The total number of lesions was counted (Fig. 5). Mice fed with a high cholesterol diet showed a dramatic increase in vascular lesions. Both MDL-72974A and aminoguanidine caused a reduction in the occurrence of fatty lesions. 


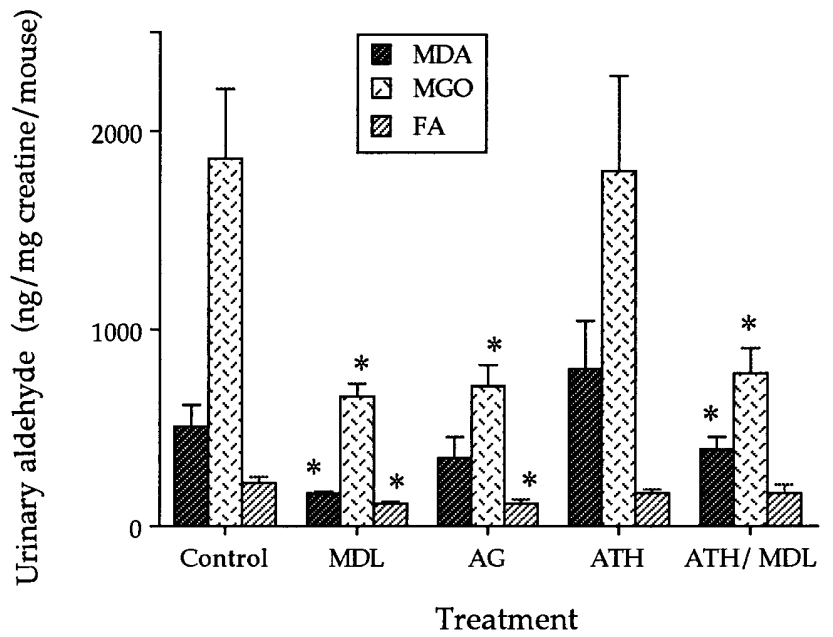

Fig. 4 Effect of MDL-72974A, aminoguanidine and atherogenic diet on urinary excretion of aldehydes in KKAy mice. Aldehydes were assessed 10 weeks after treatment. Data represent means \pm SEM of eight mice. ${ }^{*} p<0.01$ in comparison to the control group. $M D A$, malondialdehyde; $M G O$, methylglyoxal; $F A$, formaldehyde

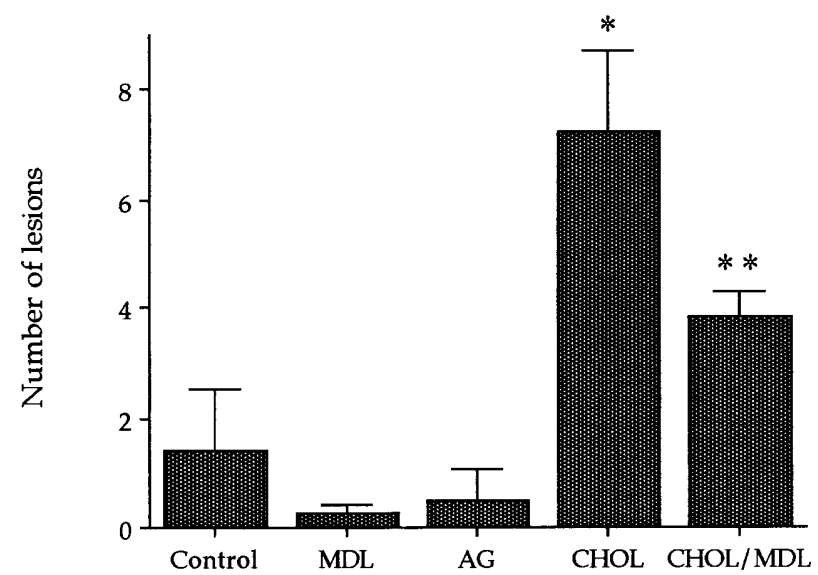

Fig. 5 Effect of MDL-72974A, aminoguanidine and atherogenic diet on the formation of atherosclerotic lesions in KKAy mice. Aortae were collected from KKAy mice after 16 weeks of treatment. Data represent means \pm SEM of eight mice. $* p<0.01$ in comparison to the control group and $* *$ in comparison to $\mathrm{CHOL}$ (mice fed with atherogenic diet without drug treatment)

\section{Discussion}

It has been proposed that increased SSAO-mediated deamination could play an important role in the initiation or development of angiopathy [16]. Both deaminated products, formaldehyde and methylglyoxal, can cross-link and thus alter functional and structural proteins. This process can initiate acute damage and subsequently trigger atherogenesis and vascular complications [17, 33]. This could explain the finding that increased serum SSAO is associated with diabetic complications $[1,2,3,6]$. It also suggests that an increase in SSAO-mediated deamination could be a risk factor for diabetic complications. Indeed an increase in SSAO activity occurs not only in diabetes, but also in heart patients and obese individuals [9]. A 5-year follow-up study has shown that the mortality of heart patients showing higher serum SSAO is more than twice that of heart patients with low SSAO activity [10]. It has been suggested that serum SSAO activity might be a useful independent prognostic marker for these patients.

KKAy mice, a well-established model for Type II diabetes, which have hyperinsulinaemia but not overt hyperglycaemia $[25,26]$, were used in this study. Atherosclerotic lesions were induced following chronic feeding of a high cholesterol diet. To substantiate the hypothesis, selective SSAO inhibitors were used to determine whether or not they could prevent the formation of atherosclerotic lesions. MDL-72974A was initially developed as a specific monoamine oxidase B (MAO-B) inhibitor, and has been studied in clinical trials for the treatment of Parkinson's disease. It has been found to be equally potent at inhibiting SSAO activity [30]. This compound is a $\beta$-fluoro monoamine mechanism-based irreversible inhibitor with very low $\mathrm{IC}_{50}$ and $\mathrm{K}_{\mathrm{I}}$ values [34]. No apparent toxicity was reported or observed after chronic administration. Aminoguanidine, a nucleophilic hydrazine derivative, is well known for its action in blocking advanced glycation [35] and it has been shown to prevent diabetic complications such as retinopathy [36], nephropathy [37] and neuropathy [38]. Although it blocks glycation, it also inhibits quinone enzymes such as SSAO [20]. In fact, it is highly potent at inhibiting SSAO activity and this enzyme is responsible for generating toxic aldehydes [20]. Aminoguanidine has been shown to show anti-atherogenic effects in cholesterol-fed rabbits [39]. This compound was therefore also used in this study as a positive control, which could prevent atherogenesis in diabetic mice.

KKAy mice possess similarly high aorta SSAO activity as do C57BL/6 mice, which is relatively vulnerable to atherosclerosis after chronic treatment with a high cholesterol diet. Our observation is consistent with the theory that high SSAO is associated with vulnerability to atherosclerosis in different strains of mice [33]. It is also interesting to note that human SSAO in different tissues is approximately tenfold higher than that in rodents [40], whereas rabbits possess tenfold higher SSAO in comparison to humans. Rabbits are well known to be one of the most vulnerable species in developing atherosclerosis.

Both MDL-72974A and aminoguanidine have been shown to be quite potent in inhibiting KKAy SSAO activity. The excretion of methylamine was substantially increased following inhibition of SSAO by these compounds. The largest increase in urinary methylamine was detected around 2 to 6 weeks after the treatment started and the methylamine gradually decreased. This suggests that a feedback mechanism could be involved, such as induction of liver microso- 
mal enzyme activities or perhaps SSAO synthesis is up-regulated, and subsequently increased metabolism of the SSAO inhibitors took place. The re-synthesis rate of SSAO is reasonably rapid with aorta SSAO activity recovering to $50 \%$ to initial levels in 2 to 3 days after complete inhibition by a single SSAO inhibitor treatment. Although aorta SSAO activity was initially completely inhibited, approximately $10 \%$ of enzyme activity could be detected after 16 weeks of treatment with the same daily dosage. This relatively small amount of SSAO is nevertheless sufficient to cause significant metabolism of methylamine.

Creatine has been shown to be a major precursor for methylamine [32]. It is therefore possible that the reduction of methylamine with time could be due to a decrease in the synthesis of creatine. Urinary creatine levels, however, did not decreased with age. The excretion of creatine was not affected by high cholesterol intake or by SSAO inhibitors. These observations suggest that the change in methylamine excretion is not due to the availability of creatine.

Albuminuria or microalbuminuria are well known useful markers for diabetic nephropathy and cardiovascular risk factors [41]. Our observation that albumin and protein excretion increased with age suggests that progressive vascular complications probably occurred in these diabetic mice. A cholesterol diet seemed to cause an unexpected reduction in albumin and protein excretions. The reason for this is not clear, although cholesterol could have altered protein binding or clearance. The SSAO inhibitor, MDL-72974A, reduced albuminuria and proteinuria in both cholesterol-treated and untreated mice. Aminoguanidine was found to ameliorate albuminuria in diabetic hypertensive rats (SHR) [42] in accordance with this previous observation. MDL-72974A is a highly selective mechanism-based amine oxidase inhibitor and it does not affect protein (i.e. RNase) glycation in vitro. The present results support the idea that a reduction in SSAO activity, and a subsequent decrease in toxic aldehydes and oxidative stress, might be responsible for the ameliorated atherogenesis process.

The potential involvement of toxic aldehydes (derived from SSAO-mediated deamination) in initiating vascular damage has been further supported by the direct measurement of aldehydes. Both formaldehyde and methylglyoxal levels were reduced after treatment with MDL-72774A or aminoguanidine. An increase in methylglyoxal has been proposed to be involved in some diabetic complications [43]. SSAO inhibitors also reduced malondialdehyde (MDA) levels, although MDA is not a deaminated product derived from SSAO-catalysed reaction. MDA is an end product of lipid oxidation and a marker of oxidative stress. Our results are consistent with several reports which also indicate that malondialdehyde is increased in diabetic patients and animals $[44,45]$. Blocking SSAO activity reduced toxic aldehydes and hydrogen perox- ide production that could have been responsible for inducing vascular damage, and therefore the reduction in malondialdehyde might be an indirect result of inhibition of SSAO activity.

Fatty streaks were detected in some obese KKAy mice, even without treatment with a high cholesterol diet. We have observed that the cholesterol levels in blood were increased more than tenfold following the feeding of the high cholesterol diet. This enhanced atherosclerotic lesions substantially. Inhibition of SSAO activity caused a reduction in such lesions. The anti-atherogenic effect of aminoguanidine is consistent with an earlier observation using a rabbit/cholesterol diet model [39]. It is not clear whether such an effect is due to blockade of glycation, since aminoguanidine is highly potent at inhibiting SSAO activity [20]. The mechanism of action of aminoguanidine in preventing glycation is further complicated by its capacity to interact with and detoxify methylglyoxal [46]. SSAO is located on the membrane surface of endothelial and vascular smooth muscle cells. Toxic aldehydes produced via SSAO-catalysed deamination cannot be readily detoxified, since aldehyde dehydrogenase is absent from serum, although the enzyme has been found in red blood cells [47]. Both formaldehyde and methylglyoxal are extremely reactive, and would probably interact quickly with proteins immediately adjacent to membrane SSAO sites where the aldehydes are produced. Therefore, proteins located on the vessel walls are most susceptible to damage, which can be the initial sites leading to atherosclerosis.

In conclusion, our study supports the hypothesis that semicarbazide-sensitive amine oxidase (SSAO), an enzyme selectively located in vascular tissues catalyses the deamination of methylamine and aminoacetone in vivo. Formaldehyde and methylglyoxal are produced respectively, as well as $\mathrm{H}_{2} \mathrm{O}_{2}$ and ammonia, which are all potentially cytotoxic. An increase in SSAO-mediated deamination could be related to atherosclerosis and diabetic complications. Figure 6 summarizes the hypothesis.

Both formaldehyde and methylglyoxal are capable of cross-linking proteins and enhancing advanced glycation. This can alter functional and structural proteins, initiate vascular damage, and cause chronic protein aggregation and deposition in blood vessels, subsequently leading to atherosclerosis. Hydrogen peroxide increases oxidative stress and is involved in atherogenesis. The deaminated products could enhance oxidation of low-density lipoprotein (LDL), which is actively involved in atherogenesis. Damage in the vascular system could cause further exposure of SSAO or leakage of intracellular SSAO. This would create a vicious cytotoxic cycle for angiopathy. Reduction of SSAO-mediated deamination might break this cycle and reduce the vascular damage associated with diabetic complications. 
Acknowledgements. The authors wish to thank the Canadian Institute of Health Research (previous MRC), Canadian Heart and Stroke Foundation and Saskatchewan Health for their continuing financial support.

\section{References}

1. Nilsson S-E, Tryding N, Tufvesson G (1968) Serum monoamine oxidase in diabetes mellitus and some other internal diseases. Acta Med Scand 184:105-108

2. Yuen C-T, Easton D, Misch K, Rhodes E (1987) Increased activity of serum amine oxidase in granuloma annulare, necrobiosis lipoidics and diabetes. Br J Dermatol 11:643649

3. Boomsma F, Meiracker A-H van den, Winkel S et al. (1999) Circulating semicarbazide-sensitive amine oxidase is raised both in type I (insulin-dependent), in type II (noninsulin-dependent) diabetes mellitus and even in childhood type I diabetes at first clinical diagnosis. Diabetologia 42:233-237

4. Hayes B-E, Clarke D-E (1990) Semicarbazide-sensitive amine oxidase activity in streptozotocin diabetic rats. Res Commun Chem Pathol Pharmacol 69:71-83

5. Elliott J, Fowden A-L, Callingham B-A, Sharman D-F, Silver M (1991) Physiological and pathological influences on sheep blood plasma amine oxidase: effect of pregnancy and experimental alloxan-induced diabetes mellitus. Res Vet Sci 50:334-339

6. Garpenstrand H, Ekblom J, Backlund L-B, Oreland L, Rosenqvist U (1999) Elevated plasma semicarbazide-sensitive amine oxidase (SSAO) activity in Type 2 diabetes mellitus complicated by retinopathy. Diabet Med 16:514521

7. Boomsma F, Veldhuisen D-J van, Kam P-J de et al. (1997) Plasma semicarbazide-sensitive amine oxidase is elevated in patients with congestive heart failure. Cardiovasc Res 33:387-391

8. Ishizaki F (1990) Plasma benzylamine oxidase activity in cerebrovascular disease. Eur Neurol 30:104-107

9. Mészáros Z, Szombathy T, Raimondi L, Karadi I, Romics L, Magyar K (1999) Elevated serum semicarbazide-sensitive amine oxidase activity in non-insulin-dependent diabetes mellitus: correlation with BMI and serum triglyceride. Metabolism 48:113-117

10. Boomsma F, De Lam P-J, Tjeerdsma G, Van den Meiracker A-H, Van Veldhuisen D-J (2000) Plasma semicarbazidesensitive amine oxidase (SSAO) is an independent prognostic marker for mortality in chronic heart failure. Eur Heart J 21:1859-1863

11. Yu P-H (2000) Semicarbazide-sensitive amine oxidase (SSAO) and mortality in chronic heart failure. Eur Heart J 21:1812-1814

12. Buffoni F (1995) Semicarbazide-sensitive amine oxidase: some biochemical properties and general consideration. Prog Brain Res 106:323-331

13. Yu P-H (1990) Deamination of aliphatic amines of different chain lengths by rat aorta semicarbazide-sensitive amine oxidase. J Pharm Pharmacol 42:882-884

14. Lyles G-A, Chalmers J (1992) The metabolism of aminoacetone to methylglyoxal by semicarbazide-sensitive amine oxidase in human umbilical artery. Biochem Pharmacol 31:1417-1424

15. Callingham B-A, Crosbie A-E, Rous B-A (1995) Some aspects of the pathophysiology of semicarbazide-sensitive amine oxidase enzymes. Prog Brain Res 106:305-321
16. Yu P-H (1998) Deamination of methylamine and angiopathy; toxicity of formaldehyde, oxidative stress and relevance to protein glycoxidation in diabetes. J Neural Transm 52 [Suppl]:201-216

17. Yu P-H, Zuo D-M (1993) Oxidative deamination of methylamine by semicarbazide-sensitive amine oxidase leads to cytotoxic damage in endothelial cells; possible consequence for diabetes. Diabetes 42:594-603

18. Deng Y-L, Boomsma F, Yu P-H (1998) Deamination of methylamine and aminoacetone increases aldehydes and oxidative stress in rats. Life Sci 63:2049-2058

19. Yu P-H, Zuo D-M (1996) Formaldehyde produced endogenously via deamination of methylamine; a potential risk factor for initiation of endothelial injury. Atherosclerosis 120:189-197

20. Yu P-H, Zuo D-M (1997) Aminoguanidine inhibits semicarbazide-sensitive amine oxidase activity; implication for advanced glycation and angiopathy in diabetes. Diabetologia 363:1243-1250

21. Jones D-J, Burnett P-C (1975) Creatine metabolism and toxicity. Kidney Int 7:S294-S298

22. Yu P-H, Dyck R-F (1998) Impairment of methylamine clearance in uremic patients and its nephropathological implications. Clin Nephrol 49:299-302

23. Siracusa L-D (1994) The agouti gene: turned on to yellow. Trends Genet 10:423-428

24. Hayase M, Yoshihiro O, Katsuura G, Shintaku H, Hosoda K, Nakao K (1996) Regulation of obese gene expression in KK mice and congenic lethal yellow obese KKAy mice. Am J Physiol 271:E333-E339

25. Iwatsuka H, Shino A, Suzuoki Z (1970) General survey of diabetic features of yellow KK mice. Endocrinol Jpn $17: 23-35$

26. Wehner H, Hohn D, Faix-Schade U, Huber H, Walzer P (1972) Glomerular changes in mice with spontaneous hereditary diabetes. Lab Invest 27:331-340

27. Deng Y-L, Yu P-H (1999) Simultaneous determination of formaldehyde and methylglyoxal in urine; involvement of deamination of methylamine in diabetic complication. J Chromatogr Sci 37:317-322

28. Tietz N-W (1982) Fundamentals of clinical chemistry. Saunders, Philadelphia, pp 994-999

29. Fielding B-A, Price D-A, Houlton C-A (1983) Enzyme immunoassay for urinary albumin. Clin Chem 29:355-357

30. Paigen B, Morrow A, Brandon C, Mitchell D, Holmes P-A (1985) Variation in susceptibility to atherosclerosis among inbred strains of mice. Atherosclerosis 57:65-73

31. Yu P-H, Zuo D-M (1991) (E)-4-Fluoro-beta-fluoroethylene benzene butamine (MDL-72974A) as a highly potent inhibitor for semicarbazide-sensitive amine oxidase from vascular tissues and serum of different species. Biochem Pharmacol 43:307-312

32. Yu P-H, Deng Y-L (2000) Potential cytotoxic effect of chronic administration of creatine, a nutrition supplement to augment athletic performance. Med Hypotheses 54:726-728

33. Yu P-H, Deng Y-L (1998) Endogenous formaldehyde as a potential factor of vulnerability of atherosclerosis: Involvement of semicarbazide-sensitive amine oxidase-mediated methylamine turnover. Atherosclerosis 140:357-363

34. Zreika M, Fozard J-R, Dudley M-W, Bey P, McDonald I-A, Palfreyman M-G (1989) MDL 72974; A potent and selective enzyme-activated irreversible inhibitor of monoamine oxidase type B with potential for use in Parkinson's disease. J Neural Transm 1:243-254

35. Brownlee M, Vlassara H, Kooney A, Ulrich P, Cerami A (1986) Aminoguanidine prevents diabetes-induced arterial protein cross-linking. Science 232:1629-1632 
36. Hammes H-P, Martin S, Federlin K, Geisen K, Brownlee M (1991) Aminoguanidine treatment inhibits the development of experimental diabetic retinopathy. Proc Natl Acad Sci USA 88:11555-11558

37. Soulis-Liparota T, Cooper M, Papazoglou D, Clarke B, Jerums G (1991) Retardation by aminoguanidine of development of albuminuria, mesangial expansion, and tissue fluorescence in streptozotocin-induced diabetic rats. Diabetes 40:1328-1334

38. Kagihashi S, Kamijo M, Baba M, Yagihashi N, Nagai K, Nagai K (1992) Effect of aminoguanidine on functional and structural in peripheral nerve of STZ-induced diabetic rats diabetes. Diabetes 41:47-52

39. Panagiotopoulos S, O'Brien R-C, Bucala R, Cooper M-E, Jerums $G$ (1998) Aminoguanidine has an anti-atherogenic effect in the cholesterol-fed rabbit. Atherosclerosis 136: $125-131$

40. Boomsma F, van-Dijk J, Bhaggoe U-M, Bouhuizen A-M, Meiracker A-H van den (2000) Variation in semicarbazidesensitive amine oxidase activity in plasma and tissues of mammals. Comp Biochem Physiol C Toxicol Pharmacol 126:69-78

41. Haffner S-M, Stern M-P, Gruber M-K, Hazuda H-P, Mitchell B-D, Patterson J-K (1990) Microalbuminuria. Po- tential marker for increased cardiovascular risk factors in nondiabetic subjects? Arteriosclerosis 10:727-731

42. Edelstein D, Brownlee M (1992) Mechanistic studies of advanced glycosylation end product inhibition by aminoguanidine. Diabetes 41:26-29

43. McLellan A-C, Phillips S-A, Thornalley P-J (1992) The assay of methylglyoxal in biological systems by derivatization with 1,2-diamino-4,5-dimethoxybenzene. Anal Biochem 206:17-23

44. Slatter D-A, Bolton C-H, Bailey A-J (2000) The importance of lipid-derived malondialdehyde in diabetes mellitus. Diabetologia 43:550-557

45. Gallaher D-D, Csallany A-S, Shoeman D-W, Olson J-M (1993) Diabetes increases excretion of urinary malonaldehyde conjugates in rats. Lipids 28:663-666

46. Lo T-W, Selwood T, Thornalley P-J (1994) The reaction of methylglyoxal with aminoguanidine under physiological conditions and prevention of methylglyoxal binding to plasma proteins. Biochem Pharmacol 48:18651870

47. Helander A, Tottmar O (1987) Metabolism of biogenic aldehydes in isolated human blood cells, platelets and in plasma. Biochem Pharmacol 36:1077-1082 